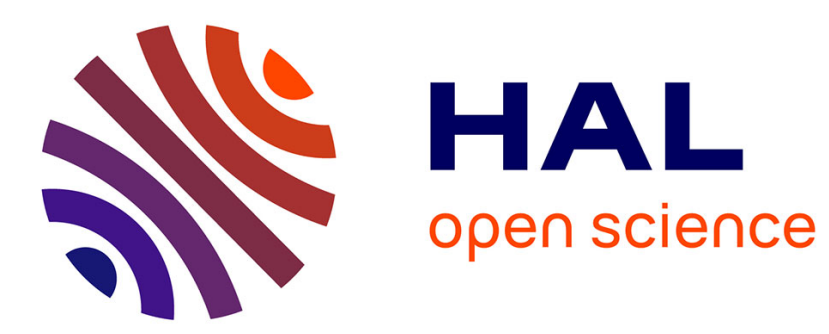

\title{
Non-linear modeling of centrifugal stiffening effects for accurate bladed component reduced-order models
}

\author{
E Khalifeh, E Piollet, A Millecamps, Alain Batailly
}

\section{To cite this version:}

E Khalifeh, E Piollet, A Millecamps, Alain Batailly. Non-linear modeling of centrifugal stiffening effects for accurate bladed component reduced-order models . ASME Turbo Expo, Jun 2017, Charlotte, United States. hal-01618313

\section{HAL Id: hal-01618313 \\ https://hal.science/hal-01618313}

Submitted on 17 Oct 2017

HAL is a multi-disciplinary open access archive for the deposit and dissemination of scientific research documents, whether they are published or not. The documents may come from teaching and research institutions in France or abroad, or from public or private research centers.
L'archive ouverte pluridisciplinaire HAL, est destinée au dépôt et à la diffusion de documents scientifiques de niveau recherche, publiés ou non, émanant des établissements d'enseignement et de recherche français ou étrangers, des laboratoires publics ou privés. 


\title{
Non-linear modeling of centrifugal stiffening effects for accurate bladed component reduced-order models
}

\author{
E. Khalifeh ${ }^{1}$, E. Piollet ${ }^{1}$, A. Millecamps ${ }^{2}$, A. Batailly ${ }^{1}$
}

\begin{abstract}
The modeling of centrifugal stiffening effects on bladed components is of primary importance in order to accurately capture their dynamics depending on the rotor angular speed. Centrifugal effects impact both the stiffness of the component and its geometry. In the context of the small perturbation framework, when considering a linear finite element model of the component, an assumption typically made in the scientific literature involves a fourth-order polynomial development of the stiffness matrix in terms of the angular speed. This polynomial development may fail to provide an accurate representation of the geometry evolution of a blade. Indeed, the error on the blade-tip displacement associated to the use of a linear finite element model quickly reaches the same order of magnitude as the blade-tip/casing clearance itself thus yielding a $100 \%$ error on the blade-tip/casing clearance configuration. This article focuses on the presentation of a methodology that allows for creating accurate reduced order models of a 3D finite element model accounting for centrifugal stiffening with a very precise description of the blade-tip/casing clearance configuration throughout a given angular speed range. The quality of the obtained reduced order model is underlined before its numerical behaviour in the context of non-linear dynamic simulations be investigated. It is evidenced that the new reduced order model features specific interactions that could not be predicted with a linear model. In addition, results highlight the limitations of numerical predictions made for high angular speeds with a linear model. Finally, a particular attention is paid to the numerical sensitivity of the proposed model. As a downside of its increased accuracy, it is underlined that its computation must be done carefully in order to avoid numerical instabilities.

Keywords

rotor/stator interaction; rubbing; nonlinear dynamics; unilateral contact dynamics; friction; abradable coating

1 - Department of Mechanical Engineering, École Polytechnique de Montréal, P.O. Box 6079, Succ. Centre-Ville, Montréal, Québec, Canada $\mathrm{H} 3 \mathrm{C}$ 3A7

2 - Safran Aircraft Engines, site de Villaroche, Moissy-Cramayel, 77550, France
\end{abstract}




\title{
Modélisation non-linéaire des effets centrifuges pour le développement de modèles réduits de composants aubagés
}

\author{
E. Khalifeh ${ }^{1}$, E. Piollet ${ }^{1}$, A. Millecamps ${ }^{2}$, A. Batailly ${ }^{1}$
}

\begin{abstract}
Résumé
La modélisation des effets centrifuges, et notamment du raidissement centrifuge, pour les composants aubagés est particulièrement importante dans l'optique de représenter précisément leur comportement dynamique dépendamment de la vitesse de rotation. Les effets centrifuges se manifestent de deux façons : (1) par une modification de la raideur de la structure et, (2) par une modification de sa géométrie. Dans le cadre de l'hypothèse des petites perturbations, lorsque des modèles éléments finis linéaires sont utilisés, une hypothèse fréquente dans la littérature scientifique est l'utilisation d'une représentation polynômiale de degré quatre de la matrice de raideur en fonction de la vitesse de rotation. Ce développement polynômial peut toutefois s'avérer insuffisant pour représenter avec précision la modification de géométrie du composant. En effet, l'erreur effectuée avec un tel développement sur le déplacement des nœuds du sommet d'aube est typiquement du même ordre de grandeur que le jeu aube/carter lui-même, impliquant une erreur de $100 \%$ sur le jeu aube/carter prédit. Cet article porte sur la présentation d'une méthodologie permettant de créer des modèles réduits précis de modèles éléments finis 3D de composants aubagés, en prenant en compte les effets centrifuges tout en assurant une description très précise des jeux aubes/carter. La qualité des résultats obtenus est démontrée dans la première section de l'article. Puis, dans une deuxième section, il est mis en évidence que cette méthodologie permet de prédire de nouvelles interactions que les modélisations linéaires usuelles ne permettent pas de prédire. Une attention particulière est portée à la sensibilité numérique de la méthodologie proposée. En contrepartie d'une précision accrue, cette méthodologie doit être mise en place avec prudence afin d'éviter de possibles instabilités numériques.

Mots-clés

interaction rotor/stator; frottement aube/carter; dynamique non-linéaire; dynamique du contact; frottement; revêtement abradable

1 - Département de génie mécanique, École Polytechnique de Montréal, P.O. Box 6079, Succ. Centre-Ville, Montréal, Québec, Canada H3C 3A7

2 - Safran Aircraft Engines, site de Villaroche, Moissy-Cramayel, 77550, France
\end{abstract}




\section{INTRODUCTION}

The ability to accurately predict complex non-linear interactions within modern aircraft engines - such as those due to unilateral contact constraints between rotating components and their static counterpart $[1,2,3,4,5]$ - is closely related to the efficient modeling of the structural components under investigation. In particular, accounting for inertial - or centrifugal - effects on bladed components remains challenging since it comes at a significant computational cost. The impact of inertial effects on bladed components is twofold: (1) the stiffness of the component changes with the angular speed $\Omega$ which yields a possibly large shift of its first eigenfrequencies and, (2) the blade geometry is modified which yields a modification of the blade-tip/casing clearance configuration. For most aircraft engine stages (fan, compressors and turbines, see Fig. 1), existing numerical strategies relying on the combination of reduced order models and a linear approximation of centrifugal effects $[6,5]$ provide an accurate representation of the component's stiffness over a wide angular speed range. However, because it relies on linear finite element models, this strategy yields an approximated calculation of the blade-tip/casing clearance [7]. Indeed, the importance of the error on the blade-tip displacement associated to the use of a linear finite element model quickly reaches the same order of magnitude as the very tight blade-tip/casing clearance itself thus possibly yielding a $100 \%$ error on the blade-tip/casing clearance configuration.

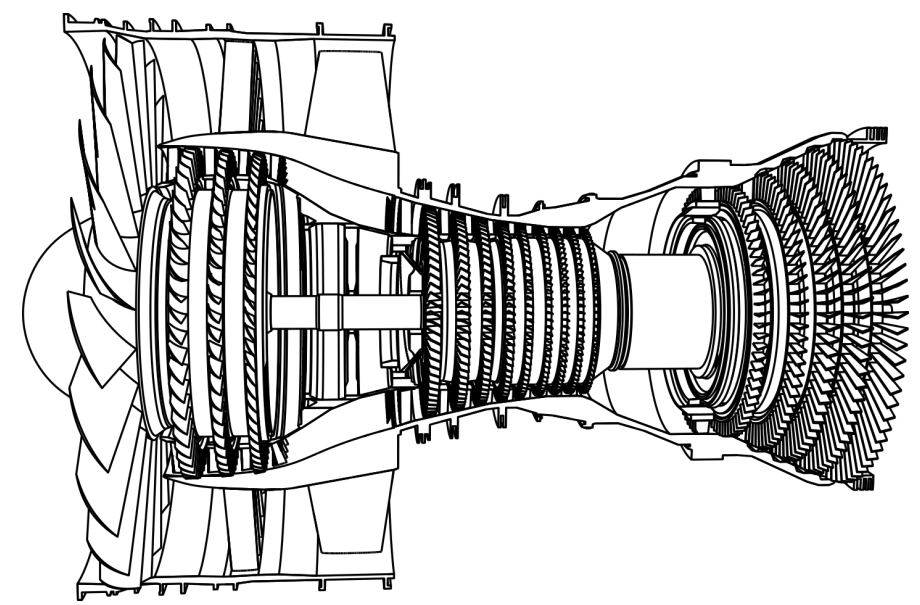

Figure 1. Schematic cut-view of an aircraft engine.

Recent numerical investigations have underlined the key role of blade-tip/casing clearances both from a structural [8] and an aerodynamic [9] standpoint. Hence, it is particularly important that numerical models be very accurate with respect to this quantity. In this article, the numerical strategy initially presented in [6] and used in [5] in combination with modal reduction and cyclic symmetry, is generalized and improved in order to obtain a much more accurate representation of blade-tip/casing clearance configuration. The limiting assumption involving a fourth-order polynomial development of the stiffness matrix in terms of the angular speed - which implies a linear computation of centrifugal effects [6] - is here replaced by a more versatile formulation consistent with a non-linear calculation of centrifugal effects. The obtained reduced-order model thus advantageously combines an enhanced modeling of its stiffness variation as a function of the angular speed $\Omega$ and a very accurate representation of the blade-tip/casing clearance configuration.

In the first section of the article, details are given with respect to the blade model used for validation of the proposed method. In the second section, the employed numerical procedure is thoroughly presented, the exact values of the coefficients for a polynomial interpolation of degree six of the blade's stiffness are given explicitly. In the next section, the focus is made on the convergence of the blade's eigenfrequencies depending on: (1) the considered reduction parameter and (2) the accuracy of the predicted blade-tip/casing clearance configuration over a wide angular speed range. Then, in the final section, the computed reduced-order model is used for the simulation of rotor/stator interactions. The obtained results are compared with those obtained with the linear formulation. 
Note: For the sake of confidentiality, all presented results have been normalized.

\section{BLADE OF INTEREST}

The proposed methodology is applicable to any bladed component of an aircraft engine or a large gas turbine. In this paper, it is applied on the finite element model of a low-pressure compressor blade, see Fig. 2. All computations carried out in this article (eigenfrequencies, blade/casing clearances, contact simulations, etc.) are based on this blade model. It should be underlined that the extension of the proposed methodology to full bladed disk structures is immediate since it is fully compatible with the developments introduced in [5].

\section{FORMULATION}

The polynomial interpolation of the stiffness matrix $\mathbf{K}(\Omega)$ presented in [6] is the basis of the employed formulation:

$\mathbf{K}(\Omega)=\sum_{p=0}^{N_{p}} \Omega^{2 p}\left[\mathbf{K}_{p}\right]$

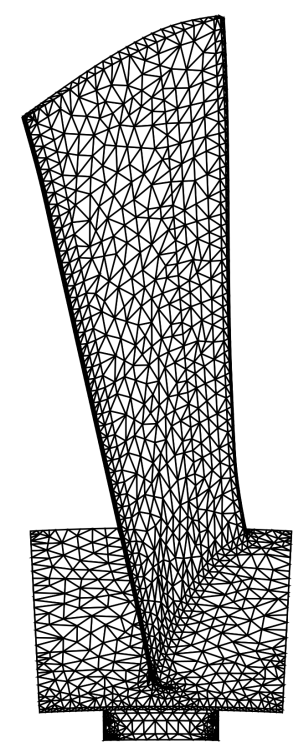

Figure 2. Lowpressure compressor blade.

This interpolation only relies on the following assumptions: (1) the angular speed $\Omega$ is constant and (2) the damping and the stiffness matrices do not depend on the excitation frequency. It is recalled in [6] that for most industrial applications, a linear computation of inertial effects is acceptable. In that case, $N_{p}=2$ which yields the usual formulation [6] used in [5]:

$\mathbf{K}(\Omega)=\mathbf{K}_{0}+\Omega^{2} \mathbf{K}_{1}+\Omega^{4} \mathbf{K}_{2}$

In the particular case where the angular speed range of interest is $\Omega \in\left[\Omega_{\mathrm{a}} ; \Omega_{\mathrm{c}}\right]$ with $\Omega_{\mathrm{a}}=0$, the matrices $\mathbf{K}_{0}, \mathbf{K}_{1}$ and $\mathbf{K}_{2}$ may be expressed as functions of the stiffness matrices obtained for $\Omega=\Omega_{\mathrm{a}}=0, \Omega=\Omega_{\mathrm{c}} / 2$ and $\Omega=\Omega_{\mathrm{c}}$ :

$$
\begin{aligned}
& \mathbf{K}_{0}=\mathbf{K}\left(\Omega_{\mathrm{a}}=0\right) \\
& \mathbf{K}_{1}=\frac{1}{3 \Omega_{\mathrm{c}}^{2}}\left[16 \mathbf{K}\left(\frac{1}{2} \Omega_{\mathrm{c}}\right)-\mathbf{K}\left(\Omega_{\mathrm{c}}\right)-15 \mathbf{K}\left(\Omega_{\mathrm{a}}=0\right)\right] \\
& \mathbf{K}_{2}=\frac{4}{3 \Omega_{\mathrm{c}}^{4}}\left[\mathbf{K}\left(\Omega_{\mathrm{c}}\right)-4 \mathbf{K}\left(\frac{1}{2} \Omega_{\mathrm{c}}\right)+3 \mathbf{K}\left(\Omega_{\mathrm{a}}=0\right)\right]
\end{aligned}
$$

When a non-linear computation of inertial effects is required however, higher values of $N_{p}$ must be considered so that the interpolation provides accurate results. Using a higher value of $N_{p}$ implies a new polynomial interpolation and thus new sets of coefficients. For the sake of brevity, only the coefficients obtained for $N_{p}=3$ are given explicitly in this article. For $N_{p}=3$, based on [6], the polynomial development of the stiffness matrix is:

$\mathbf{K}(\Omega)=\tilde{\mathbf{K}}_{0}+\Omega^{2} \tilde{\mathbf{K}}_{1}+\Omega^{4} \tilde{\mathbf{K}}_{2}+\Omega^{6} \tilde{\mathbf{K}}_{3}$

with $\Omega \in\left[\Omega_{\mathrm{a}} ; \Omega_{\mathrm{c}}\right]$ and:

$$
\begin{aligned}
& \tilde{\mathbf{K}}_{0}=\frac{{\Omega_{1}}^{2} \Omega_{2}{ }^{2} \Omega_{\mathrm{c}}{ }^{2}}{\left(\Omega_{1}{ }^{2}-\Omega_{\mathrm{a}}{ }^{2}\right)\left({\Omega_{2}}^{2}-\Omega_{\mathrm{a}}{ }^{2}\right)\left({\Omega_{\mathrm{c}}}^{2}-{\Omega_{\mathrm{a}}}^{2}\right)}\left[\tilde{\mathbf{K}}\left(\Omega_{\mathrm{a}}\right)\right] \\
& -\frac{\Omega_{\mathrm{a}}{ }^{2}{\Omega_{2}}^{2} \Omega_{\mathrm{c}}{ }^{2}}{\left({\Omega_{1}}^{2}-\Omega_{\mathrm{a}}{ }^{2}\right)\left({\Omega_{2}}^{2}-{\Omega_{1}}^{2}\right)\left({\Omega_{\mathrm{c}}}^{2}-\Omega_{1}{ }^{2}\right)}\left[\tilde{\mathbf{K}}\left(\Omega_{1}\right)\right] \\
& +\frac{\Omega_{\mathrm{a}}{ }^{2} \Omega_{1}{ }^{2} \Omega_{\mathrm{c}}{ }^{2}}{\left({\Omega_{2}}^{2}-\Omega_{\mathrm{a}}{ }^{2}\right)\left({\Omega_{2}}^{2}-{\Omega_{1}}^{2}\right)\left({\Omega_{\mathrm{c}}}^{2}-{\Omega_{2}}^{2}\right)}\left[\tilde{\mathbf{K}}\left(\Omega_{2}\right)\right] \\
& -\frac{\Omega_{\mathrm{a}}{ }^{2} \Omega_{1}{ }^{2} \Omega_{2}{ }^{2}}{\left({\Omega_{\mathrm{c}}}^{2}-\Omega_{\mathrm{a}}{ }^{2}\right)\left({\Omega_{\mathrm{c}}}^{2}-{\Omega_{1}}^{2}\right)\left({\Omega_{\mathrm{c}}}^{2}-{\Omega_{2}}^{2}\right)}\left[\tilde{\mathbf{K}}\left(\Omega_{\mathrm{c}}\right)\right]
\end{aligned}
$$




$$
\begin{aligned}
& \tilde{\mathbf{K}}_{1}=-\frac{\Omega_{2}{ }^{2} \Omega_{\mathrm{c}}{ }^{2}+\Omega_{1}{ }^{2} \Omega_{\mathrm{c}}{ }^{2}+\Omega_{1}{ }^{2} \Omega_{2}{ }^{2}}{\left(\Omega_{1}{ }^{2}-\Omega_{\mathrm{a}}{ }^{2}\right)\left(\Omega_{2}{ }^{2}-\Omega_{\mathrm{a}}{ }^{2}\right)\left(\Omega_{\mathrm{c}}{ }^{2}-\Omega_{\mathrm{a}}{ }^{2}\right)}\left[\tilde{\mathbf{K}}\left(\Omega_{\mathrm{a}}\right)\right] \\
& +\frac{\Omega_{2}{ }^{2} \Omega_{\mathrm{c}}{ }^{2}+\Omega_{\mathrm{a}}{ }^{2} \Omega_{\mathrm{c}}{ }^{2}+\Omega_{\mathrm{a}}{ }^{2} \Omega_{2}{ }^{2}}{\left(\Omega_{1}{ }^{2}-\Omega_{\mathrm{a}}{ }^{2}\right)\left(\Omega_{2}{ }^{2}-\Omega_{1}{ }^{2}\right)\left(\Omega_{\mathrm{c}}{ }^{2}-\Omega_{1}{ }^{2}\right)}\left[\tilde{\mathbf{K}}\left(\Omega_{1}\right)\right] \\
& -\frac{\Omega_{1}{ }^{2} \Omega_{\mathrm{c}}{ }^{2}+\Omega_{\mathrm{a}}{ }^{2} \Omega_{\mathrm{c}}{ }^{2}+\Omega_{\mathrm{a}}{ }^{2} \Omega_{1}{ }^{2}}{\left(\Omega_{2}{ }^{2}-\Omega_{\mathrm{a}}{ }^{2}\right)\left(\Omega_{2}{ }^{2}-\Omega_{1}{ }^{2}\right)\left(\Omega_{\mathrm{c}}{ }^{2}-\Omega_{2}{ }^{2}\right)}\left[\tilde{\mathbf{K}}\left(\Omega_{2}\right)\right] \\
& +\frac{\Omega_{1}{ }^{2} \Omega_{2}{ }^{2}+\Omega_{\mathrm{a}}{ }^{2} \Omega_{2}{ }^{2}+\Omega_{\mathrm{a}}{ }^{2} \Omega_{1}{ }^{2}}{\left(\Omega_{\mathrm{c}}{ }^{2}-\Omega_{\mathrm{a}}{ }^{2}\right)\left(\Omega_{\mathrm{c}}{ }^{2}-\Omega_{1}{ }^{2}\right)\left(\Omega_{\mathrm{c}}{ }^{2}-\Omega_{2}{ }^{2}\right)}\left[\tilde{\mathbf{K}}\left(\Omega_{\mathrm{c}}\right)\right] \\
& \tilde{\mathbf{K}}_{2}=\frac{\Omega_{\mathrm{c}}{ }^{2}+\Omega_{2}{ }^{2}+\Omega_{1}{ }^{2}}{\left(\Omega_{1}{ }^{2}-\Omega_{\mathrm{a}}{ }^{2}\right)\left(\Omega_{2}{ }^{2}-\Omega_{\mathrm{a}}{ }^{2}\right)\left({\Omega_{\mathrm{c}}}^{2}-\Omega_{\mathrm{a}}{ }^{2}\right)}\left[\tilde{\mathbf{K}}\left(\Omega_{\mathrm{a}}\right)\right] \\
& -\frac{\Omega_{\mathrm{c}}{ }^{2}+{\Omega_{2}}^{2}+\Omega_{\mathrm{a}}{ }^{2}}{\left(\Omega_{1}{ }^{2}-\Omega_{\mathrm{a}}{ }^{2}\right)\left({\Omega_{2}}^{2}-\Omega_{1}{ }^{2}\right)\left({\Omega_{\mathrm{c}}}^{2}-\Omega_{1}{ }^{2}\right)}\left[\tilde{\mathbf{K}}\left(\Omega_{1}\right)\right] \\
& +\frac{\Omega_{\mathrm{c}}{ }^{2}+\Omega_{1}{ }^{2}+\Omega_{\mathrm{a}}{ }^{2}}{\left({\Omega_{2}}^{2}-\Omega_{\mathrm{a}}{ }^{2}\right)\left(\Omega_{2}{ }^{2}-\Omega_{1}{ }^{2}\right)\left(\Omega_{3}{ }^{2}-{\Omega_{2}}^{2}\right)}\left[\tilde{\mathbf{K}}\left(\Omega_{2}\right)\right] \\
& -\frac{\Omega_{2}{ }^{2}+\Omega_{1}{ }^{2}+\Omega_{\mathrm{a}}{ }^{2}}{\left(\Omega_{\mathrm{c}}{ }^{2}-\Omega_{\mathrm{a}}{ }^{2}\right)\left(\Omega_{\mathrm{c}}{ }^{2}-\Omega_{1}{ }^{2}\right)\left({\Omega_{\mathrm{c}}}^{2}-\Omega_{2}{ }^{2}\right)}\left[\tilde{\mathbf{K}}\left(\Omega_{\mathrm{c}}\right)\right] \\
& \tilde{\mathbf{K}}_{3}=-\frac{1}{\left(\Omega_{1}{ }^{2}-\Omega_{\mathrm{a}}{ }^{2}\right)\left(\Omega_{2}{ }^{2}-\Omega_{\mathrm{a}}{ }^{2}\right)\left(\Omega_{\mathrm{c}}{ }^{2}-\Omega_{\mathrm{a}}{ }^{2}\right)}\left[\tilde{\mathbf{K}}\left(\Omega_{\mathrm{a}}\right)\right] \\
& +\frac{1}{\left(\Omega_{1}{ }^{2}-\Omega_{\mathrm{a}}{ }^{2}\right)\left({\Omega_{2}}^{2}-\Omega_{1}{ }^{2}\right)\left(\Omega_{\mathrm{c}}{ }^{2}-\Omega_{1}{ }^{2}\right)}\left[\tilde{\mathbf{K}}\left(\Omega_{1}\right)\right] \\
& -\frac{1}{\left(\Omega_{2}{ }^{2}-\Omega_{\mathrm{a}}{ }^{2}\right)\left(\Omega_{2}{ }^{2}-\Omega_{1}{ }^{2}\right)\left(\Omega_{\mathrm{c}}{ }^{2}-\Omega_{2}{ }^{2}\right)}\left[\tilde{\mathbf{K}}\left(\Omega_{2}\right)\right] \\
& +\frac{1}{\left(\Omega_{\mathrm{c}}{ }^{2}-\Omega_{\mathrm{a}}{ }^{2}\right)\left(\Omega_{\mathrm{c}}{ }^{2}-\Omega_{1}{ }^{2}\right)\left({\Omega_{\mathrm{c}}}^{2}-\Omega_{2}{ }^{2}\right)}\left[\tilde{\mathbf{K}}\left(\Omega_{\mathrm{c}}\right)\right]
\end{aligned}
$$

where:

$\Omega_{\mathrm{a}}<\Omega_{1}<\Omega_{2}<\Omega_{\mathrm{c}}$

It should be underlined that the fundamental difference between Eqs. (2) and (4) consists in the nature of the stiffness matrices $\mathbf{K}\left(\Omega_{i}\right)$. While $\mathbf{K}\left(\Omega_{i}\right)$ stems from linear finite element computations; in Eq. (4), $\tilde{\mathbf{K}}\left(\Omega_{i}\right)$ is obtained from a static non-linear computation of the application of centrifugal effects.

In order to build numerically efficient models, the polynomial interpolations defined in Eqs. (2) and (4) may be projected into a suitable subspace using the Craig-Bampton method [10]. This reduction procedure, already presented in [5] is used here but not recalled for the sake of brevity. The reduction parameter $\eta$, which controls the number of constraint modes computed at each interpolation point, is set to $\eta=10$. As a summary, key aspects of the proposed formulation - hereafter referred to as NLC-ROM (Non-Linear Centrifugal computation of inertial effects combined with a Reduced-Order Model) - are recalled in Tab. 1.

\section{CONVERGENCE}

\section{Eigenfrequencies}

Prior to any numerical investigation, it must be checked that the proposed formulation converges as the value of $N_{p}$ is increased. The eigenfrequencies of a reduced-order model

\footnotetext{
${ }^{1}$ The actual dimension of both the ROM and the NLC-ROM may be slightly lower than $3 \eta$ or $\left(N_{p}+1\right) \eta$ due to an orthonormalization of the component modes that may lead to the elimination of linearly dependent vectors.
} 


\begin{tabular}{|l|c|c|}
\hline & ROM [5] & NLC-ROM \\
\hline centrifugal effects & $\begin{array}{c}\text { linear } \\
\text { computation }\end{array}$ & $\begin{array}{c}\text { non-linear } \\
\text { computation }\end{array}$ \\
\hline $\begin{array}{l}\text { reduction } \\
\text { technique }\end{array}$ & \multicolumn{2}{|c|}{ Craig-Bampton } \\
\hline value of $N_{p}$ & $N_{p}=2$ & $N_{p} \geq 2$ \\
\hline $\begin{array}{l}\text { ROM dimension } \\
\text { depending on } \eta^{1}\end{array}$ & $\simeq 3 \eta$ & $\simeq\left(N_{p}+1\right) \eta$ \\
\hline
\end{tabular}

Table 1. Differences between the reduced order models (ROM) computed in [5] and in this article.

computed with different values of $N_{p}$ and a non-linear representation of centrifugal effectsa NLC-ROM - are first compared with the eigenfrequencies of the usual reduced-order model - referred to as ROM - computed with a linear representation of centrifugal effects. This comparison is first carried out over a wide angular speed range $\Omega \in\left[\Omega_{\mathrm{a}} ; \Omega_{\mathrm{c}}\right]$ with $\Delta \Omega=\Omega_{\mathrm{c}}-\Omega_{\mathrm{a}}=3000 \mathrm{RPM}$, see Fig. 3 .

The graphs plotted in Fig. 3 focus on the first two eigenfrequencies of the blade model (which correspond to the first bending and torsion modes, namely $1 \mathrm{~B}$ and $1 \mathrm{~T}$ modes). They emphasize that the proposed formulation converges as $N_{p}$ increases. In particular, numerical oscillations of the eigenfrequencies that appear for a too low degree of the polynomial interpolation vanish when $N_{p}>4$. Also, it appears clearly in Fig. 3 that there is a smallyet significant - gap between the eigenfrequencies obtained with a converged NLC-ROM (-) and the eigenfrequencies obtained with a ROM (-). The same gap is observed between the finite element reference solution of each model which underlines that it originates from the different modeling of inertial effects. For the investigated blade model, a linear computation of centrifugal effects yields an overestimation (about 2 to $5 \%$ ) of the first bending mode eigenfrequency and an underestimation (about 2 to $3 \%$ ) of the first torsion mode.

Similar quantities are also measured for NLC-ROM computed over a smaller angular speed range $\Omega \in\left[\Omega_{\mathrm{b}} ; \Omega_{\mathrm{c}}\right]$ with $\Omega_{\mathrm{b}}>\Omega_{\mathrm{a}}$ and $\Delta \Omega=\Omega_{\mathrm{c}}-\Omega_{\mathrm{b}}=1000$ RPM, results are

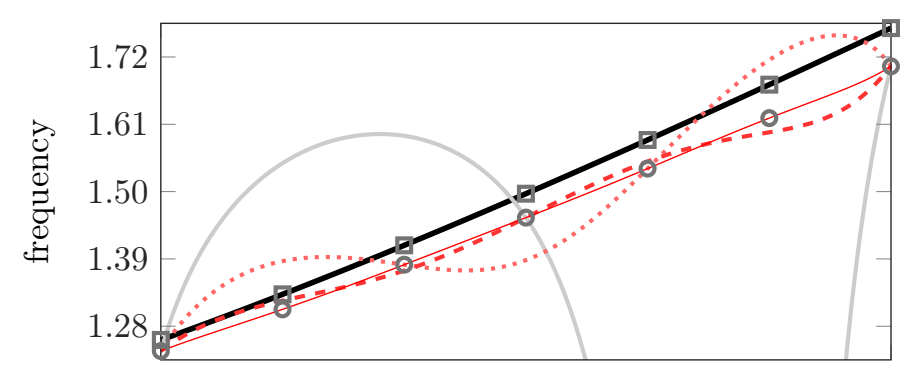

(a) mode $1 \mathrm{~B}$

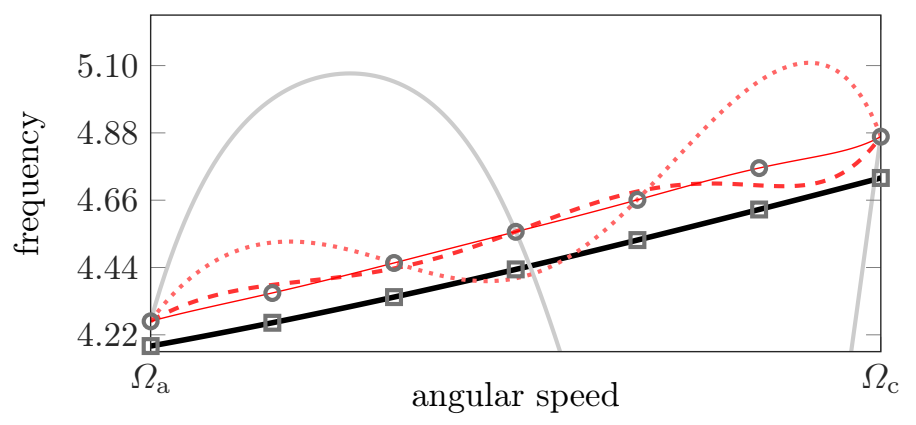

(b) mode $1 \mathrm{~T}$

Figure 3. Eigenfrequencies of the blade model over $\Omega \in\left[\Omega_{\mathrm{a}} ; \Omega_{\mathrm{c}}\right]$ for the linear model ( - ) and the non-linear models with $N_{p}=2(\square), N_{p}=3(\cdots \cdots), N_{p}=4(---)$ and $N_{p}=6(\square)$. Non-linear (०) and linear ( $\square$ ) finite element reference solutions are also plotted for comparison. 
depicted in Fig. 4. Because the angular speed range is narrower, it is found that numerical oscillations of the eigenfrequencies are significantly reduced, even for low values of $N_{p}$. As a matter of fact, $N_{p}=3$ provides a very accurate approximation of the first eigenfrequencies.

In any case, this first convergence analysis suggests that $N_{p}$ should be sufficiently large in order to obtain an accurate approximation of the blade eigenfrequencies.

\section{Blade-tip/casing clearance configuration}

As mentioned in the introduction, one of the motivations for the development of the NLC-ROM is to obtain a much more accurate description of the blade-tip/casing clearance configuration, meaning a more accurate description of blade/casing clearances. The clearance variation at the leading edge due to centrifugal effects is depicted in Fig. 5 for a ROM and NLC-ROM featuring different values of $N_{p}$. The same quantity observed at the trailing edge of the investigated blade is pictured in Fig. 6 .

Both figures underline that over the angular speed range $\Omega \in\left[\Omega_{\mathrm{a}} ; \Omega_{\mathrm{c}}\right]$, ROM and NLC-ROM lead to very distinct clearance variations. The good approximation obtained with a ROM only stands for low angular speeds $\Omega<\Omega_{\mathrm{a}}$, for $\Omega>\Omega_{\mathrm{a}}$, the error on the computation of clearance variation with a usual ROM becomes even larger than typical blade/casing nominal clearances. This observation is inherent to the way centrifugal effects are accounted for since the ROM and the NLC-ROM respectively match the linear and non-linear finite element reference solutions.

Contrary to the eigenfrequencies, it is found that the value of $N_{p}$ has low to no influence over the quality of the results: the difference between clearance variations for $N_{p}=3$ and $N_{p}=6$ is about 300 times lower than the error between a ROM and a NLC-ROM. Thus, this second convergence analysis reveals that for a NLC-ROM, $N_{p}$ has low to no impact Rumerical stability of the predicted blade/casing clearance configuration.

One of the key aspects of a reduced-order model dedicated to complex non-linear structural simulations is the conditioning of the reduced structural matrices. In particular, obtaining accurate and relevant results precludes ill-conditioned matrices.

In order to ensure that reduced structural matrices are not ill-conditioned, the reduction strategy proposed in [5] relies on a fixed-interface component mode synthesis method. As

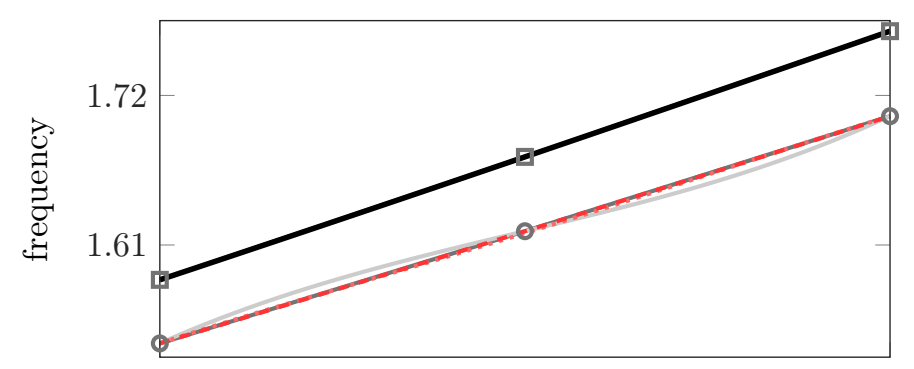

(a) mode $1 \mathrm{~B}$

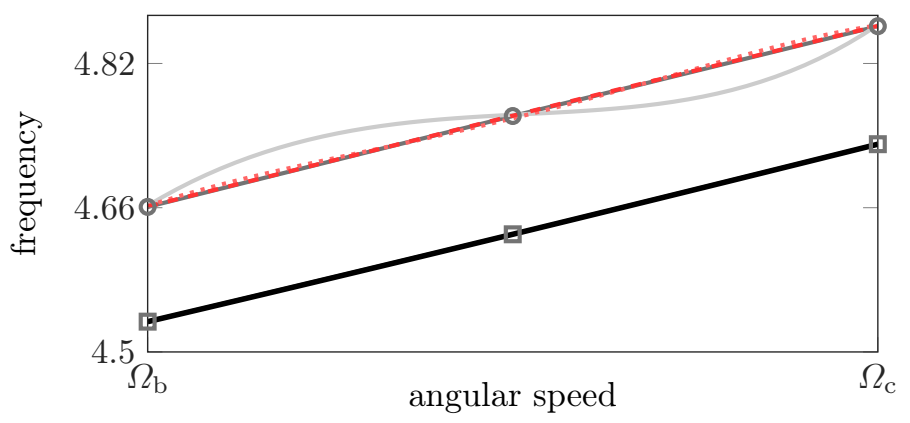

(b) mode $1 \mathrm{~T}$

Figure 4. Eigenfrequencies of the blade model over $\Omega \in\left[\Omega_{\mathrm{b}} ; \Omega_{\mathrm{c}}\right]$ for the linear model ( $\longrightarrow$ ) and the non-linear models with $N_{p}=2(-), N_{p}=3(\ldots .$.$) ) and N_{p}=4(--)^{\prime}$. Non-linear (o) and linear ( $\square$ ) finite element reference solutions are also plotted for comparison. 


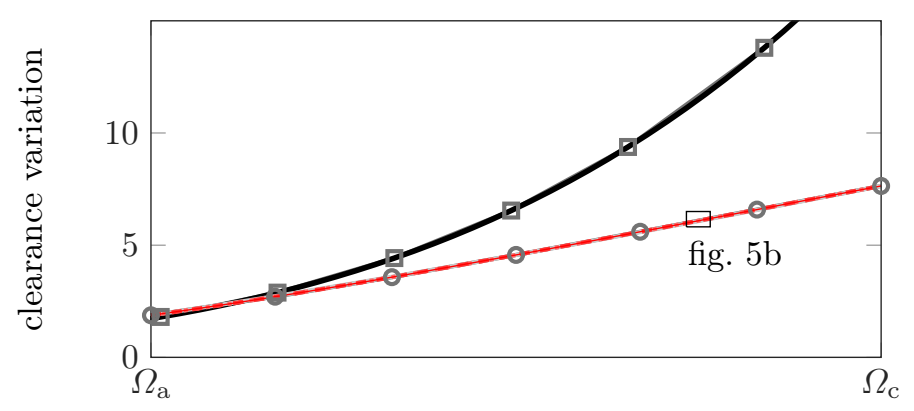

(a) view over $\left[\Omega_{a} ; \Omega_{c}\right]$

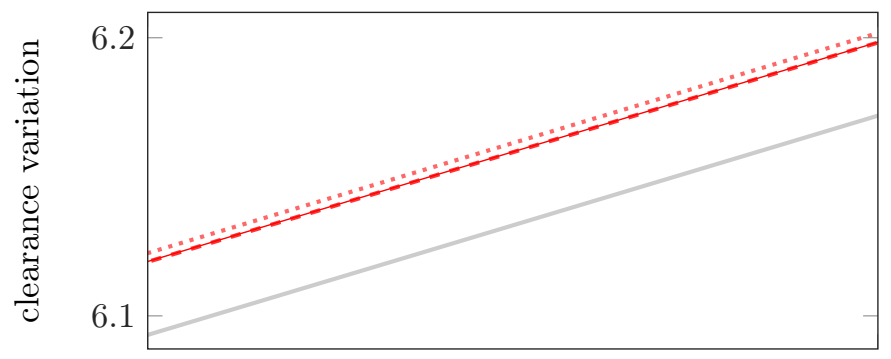

angular speed

(b) zoom

Figure 5. Clearance variation at the blade leading edge over $\Omega \in\left[\Omega_{\mathrm{a}} ; \Omega_{\mathrm{c}}\right]$ for a ROM ( $\longrightarrow$ ) and NLC-ROM with $N_{p}=2(-), N_{p}=3(\cdots \cdots), N_{p}=4\left(--_{--}\right)$and $N_{p}=6(-)$. Non-linear (o) and linear ( $\square$ ) finite element reference solutions are also plotted for comparison.

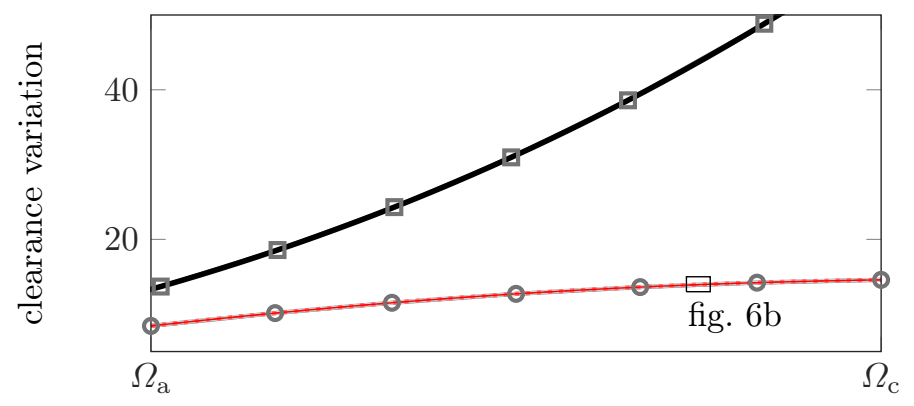

(a) view over $\left[\Omega_{a} ; \Omega_{c}\right]$

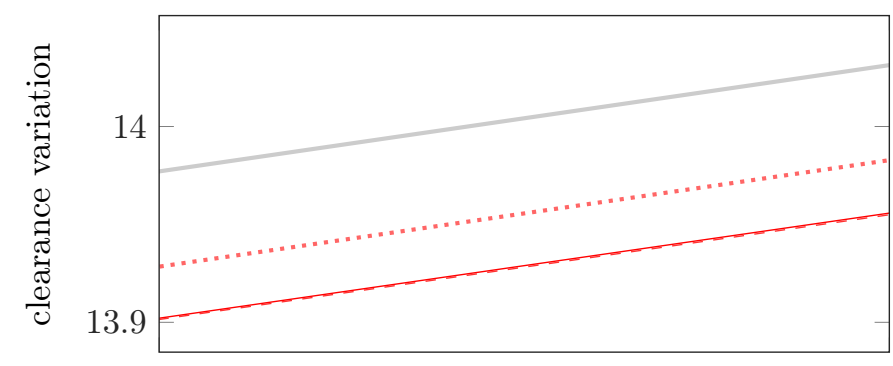

angular speed

(b) zoom

Figure 6. Clearance variation at the blade trailing edge over $\Omega \in\left[\Omega_{\mathrm{a}} ; \Omega_{\mathrm{c}}\right]$ for the linear model ( - ) and the non-linear models with $N_{p}=2(-), N_{p}=3(\cdots \cdots), N_{p}=4\left(-\Omega_{-}\right)$and $N_{p}=6(-)$. Non-linear (O) and linear ( $\square$ ) finite element reference solutions are also plotted for comparison.

an advantage towards free-interface methods, fixed-interface methods are known to be less prone to numerical sensitivity [11]. Nevertheless, the obtention of a NLC-ROM requires the 
computation of several sets of component modes: both constraint modes ${ }^{2}$ and static modes $^{3}$ must be computed for each interpolation point as underlined in Tab. 1.

For the sake of understanding numerical issues that may arise depending on the reduction basis, let us consider a limit case where $N_{p}$ is very large. For such configuration, as mentioned in Tab. 1 , there are $\left(N_{p}+1\right) \eta$ component modes. The reduction matrix $\boldsymbol{\Psi}$ would thus contain $n$ lines ( $n$ is the dimension of the finite element model) and $\left(N_{p}+1\right) \eta$ columns. Because all the component modes are related to low-frequency constraint modes of the blade model, it is very likely that the span of these component modes will be lower than their numbers thus yielding a rank deficient reduction matrix. While an orthornormalization procedure of the component modes may correct the rank deficiency, it has been observed that the reduced structural matrices may still be ill-conditioned.

In the end, the reduction strategy employed in order to get accurate NLC-ROM imposes that the value of $N_{p}$ should not be too high so that: (1) the NLC-ROM remains computationally efficient and, (2) reduced structural matrices are not ill-conditioned.

\section{Partial conclusion}

In conclusion of the convergence analysis carried out in the previous sections, a compromise must be found: on one hand large values of $N_{p}$ will favor an accurate description of the blade eigenfrequencies. On the other hand, because the polynomial interpolation is projected into a lower-dimension subspace, $N_{p}$ must not be too high in order to avoid numerical issues.

The adopted solution relies on the computation of a NLC-ROM over a limited angular speed range $\Delta \Omega$. In the following, all NLC-ROMs are computed with $\Delta \Omega=1000 \mathrm{RPM}$ and $N_{p}=3$. As underlined in Fig. 4, such parameters allow for a very accurate description of the blade eigenfrequencies. While specific results are not provided for the sake of brevity, these parameters also provide an excellent description of the blade/casing clearance configuration.

As an alternative to the proposed compromise between $N_{p}$ and the width of the considered angular speed range, one may mention the possibility to carry out a specific mathematical treatment of the reduction basis. Dedicated techniques are available in the literature [12]. Such investigations go beyond the scope of the article but constitute promising avenues in case a much wider angular speed range would have to be considered.

\section{MODAL ANALYSIS}

In this section, the free-vibration modes obtained with a NLC-ROM are compared with those obtained with a ROM by means of the Modal Assurance Criterion (MAC) [13]. The $i^{\text {th }}$ free-vibration mode computed for the ROM and the NLC-ROM are respectively denoted $\boldsymbol{\Phi}_{i}$ and $\tilde{\boldsymbol{\Phi}}_{i}$, both vectors are expressed in the same initial finite element space for the sake of comparison. MAC values are computed by comparing the first ten free-vibration modes of each reduced model to one another:

$\mathrm{MAC}_{i j}=\frac{\left(\boldsymbol{\Phi}_{i}^{\top} \tilde{\boldsymbol{\Phi}}_{j}\right)^{2}}{\left(\boldsymbol{\Phi}_{i}^{\top} \boldsymbol{\Phi}_{i}\right)\left(\tilde{\boldsymbol{\Phi}}_{j}^{\top} \tilde{\boldsymbol{\Phi}}_{j}\right)}, \quad i, j=1, \ldots 10$

As mentioned above, both the ROM and NLC-ROM are computed with a Craig-Bampton reduction parameter $\eta=10$. Since the associated reduction basis does not explicitly contain free-vibration modes of the full system, it is expected that the number of free-vibration modes accurately captured by the ROM or NLC-ROM is inferior to $\eta$ (typically 7 or 8 if $\eta=10)$. Consequently, in order to avoid any misleading comparison, the scope of our analysis will be here limited to the first five MAC values $(i \leq 5$ and $j \leq 5)$.

ROM and NLC-ROM are compared over a very large angular speed range : $\Omega \in$ $\left[0 ; 1.7 \times \Omega_{c}\right]$, MAC values are computed for $\Omega=0, \Omega_{a}, \Omega_{c}$ and $1.7 \times \Omega_{c}$. Accordingly, four NLC-ROM are computed to carry out this comparison, one for each of the following angular speed ranges: $[0 ; 1000] \mathrm{RPM},\left[\Omega_{a} ; \Omega_{a}+1000 \mathrm{RPM}\right],\left[\Omega_{c}-1000 \mathrm{RPM} ; \Omega_{c}\right]$, and $\left[1.7 \times \Omega_{c}-1000 \mathrm{RPM} ; 1.7 \times \Omega_{c}\right]$. MAC values plotted in Figs. 7 and 8 are respective to each of these NLC-ROMs.

\footnotetext{
${ }^{2}$ Constraint modes are free-vibration modes of the investigated system featuring clamped boundaries.

${ }^{3}$ Static modes are specific static deformed shapes of the investigated system.
} 


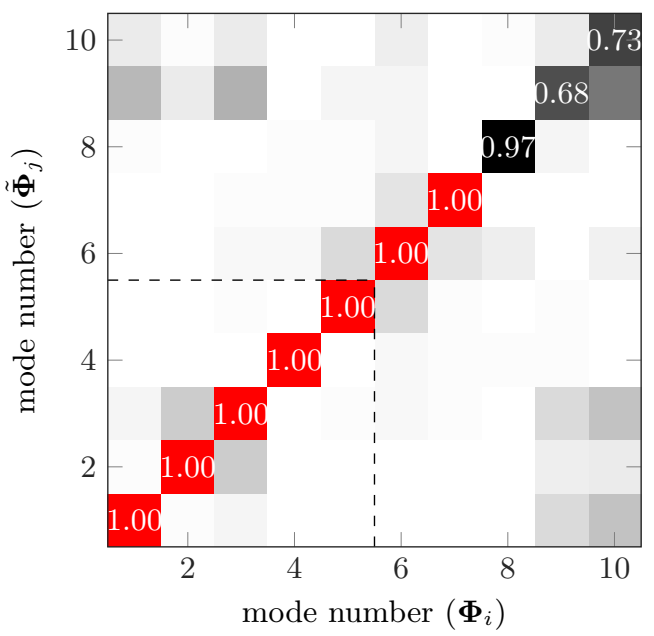

(a) $\Omega=0$

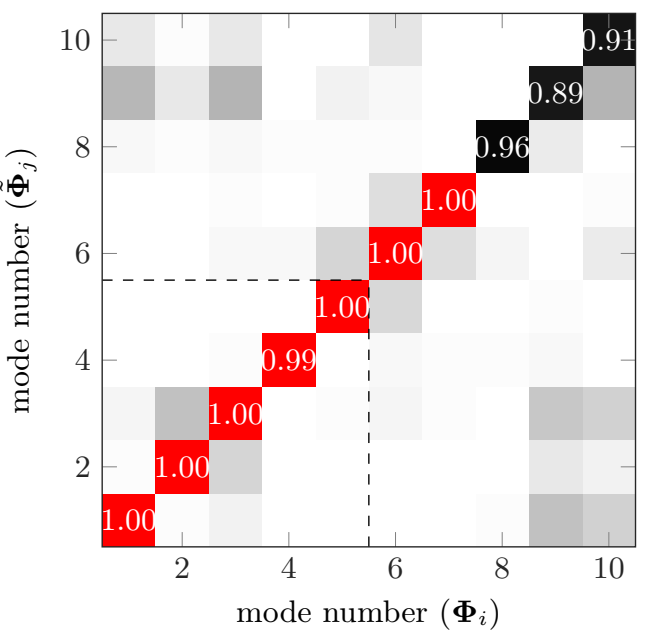

(b) $\Omega=\Omega_{\text {a }}$

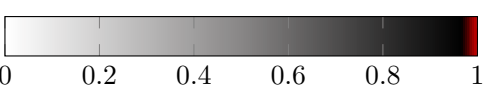

(c) colour code used for the MAC values

Figure 7. MAC of the first ten free vibration modes of a NLC-ROM with respect to those of a ROM $\left(\Omega=0\right.$ and $\left.\Omega=\Omega_{a}\right)$.

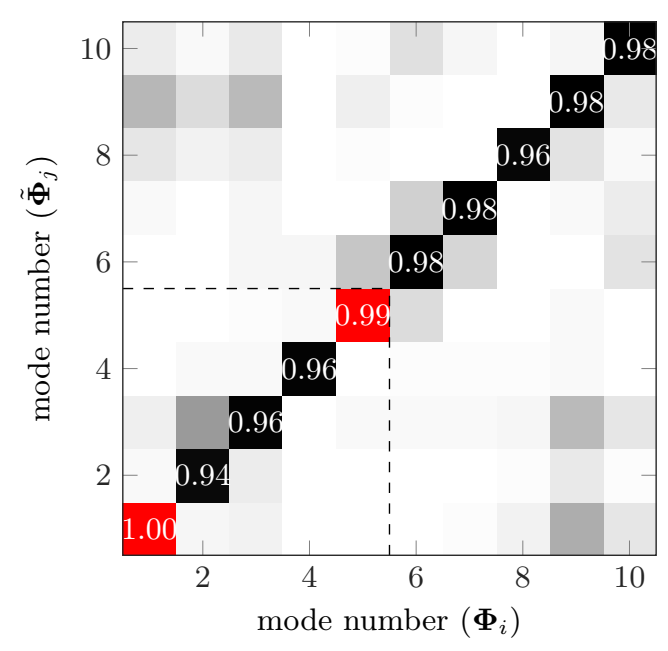

(a) $\Omega=\Omega_{\mathrm{c}}$

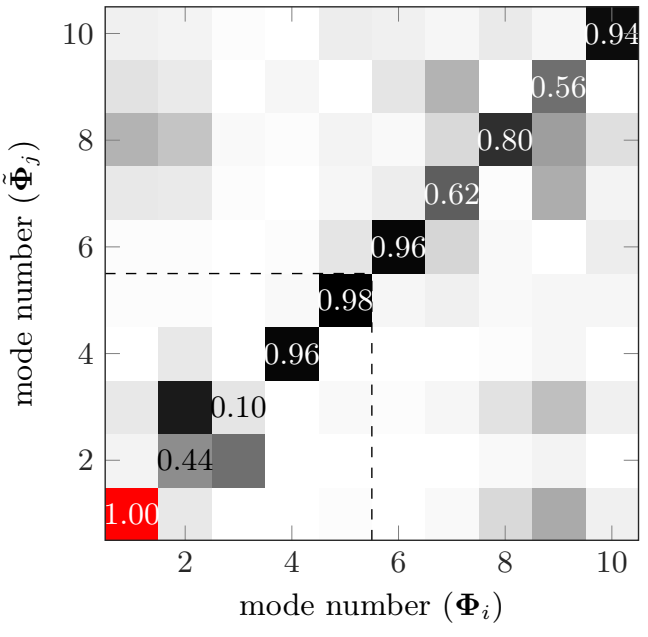

(b) $\Omega=1.7 \Omega_{\mathrm{c}}$

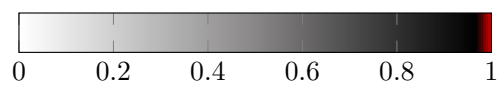

(c) colour code used for the MAC values

Figure 8. MAC of the first ten free vibration modes of a NLC-ROM with respect to those of a ROM $\left(\Omega=\Omega_{c}\right.$ and $\left.\Omega=1.7 \times \Omega_{c}\right)$.

Figures 7 and 8 underline the very good agreement between modal shapes obtained by the ROM and the NLC-ROM for $\Omega=0$ (which is obvious since inertial effects are not applied) and $\Omega=\Omega_{a}$. For higher angular speeds such as $\Omega_{c}$ and $1.7 \times \Omega_{c}$, non-negligible differences arise between the ROM and the NLC-ROM. This is patent for modes 2 and 3 (respectively the first and second torsional modes of the blade). The angular speed range over which ROM and NLC-ROM feature similar mode shapes is highly dependent on the considered blade model. As a reminder, the investigated blade belongs to the low-pressure 
compressor of an aircraft engine. It is expected that this domain would significantly shrink if fan blades were to be considered. For such blade, it is expected that a NLC-ROM would be particularly efficient. To the contrary, this domain would probably expand in the case of stiffer high-pressure compressor blades for which a classical ROM may be sufficient.

\section{CASE STUDY}

Going beyond the convergence analysis and the comparison between linear and non-linear formulations, the NLC-ROM is here employed for the simulation of blade/casing structural interactions accounting for abradable coating removal. Simulations are carried out with a dedicated computational tool developed for Safran Aircraft Engines [14].

\section{Interaction scenario}

A rubbing interaction between the low-pressure compressor blade pictured in Fig. 2 and the surrounding abradable coating is simulated over the angular speed range $\Omega \in\left[\Omega_{a}-\right.$ $\left.1000 \mathrm{RPM} ; \Omega_{a}\right]$. The abradable layer deposited on the casing is $5 \mathrm{~mm}$ thick, the friction coefficient is $\mu=0.15$, blade-tip/casing clearances at rest are identical from the blade leading edge to the trailing edge and set to $1 \mathrm{~mm}$. Numerical simulations are carried out assuming the angular speed $\Omega$ is constant. In total, 100 simulations are performed to accurately cover the considered angular speed range. Each simulation features 50 blade revolutions in a slightly deformed casing (two symmetric bumps are created) in order to initiate contact.

\section{Convergence}

Results obtained with a NLC-ROM are compared for different time integration steps: $h=10^{-7} \mathrm{~s}, h=5 \cdot 10^{-8} \mathrm{~s}$, and $h=2.5 \cdot 10^{-8} \mathrm{~s}$. A zoom over displacements and contact forces obtained for each of these time steps is ictured in Fig. 9 Both types of quantities

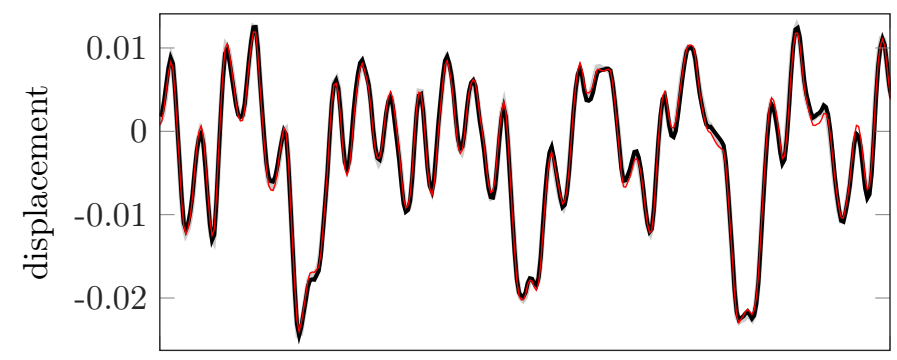

(a) displacement

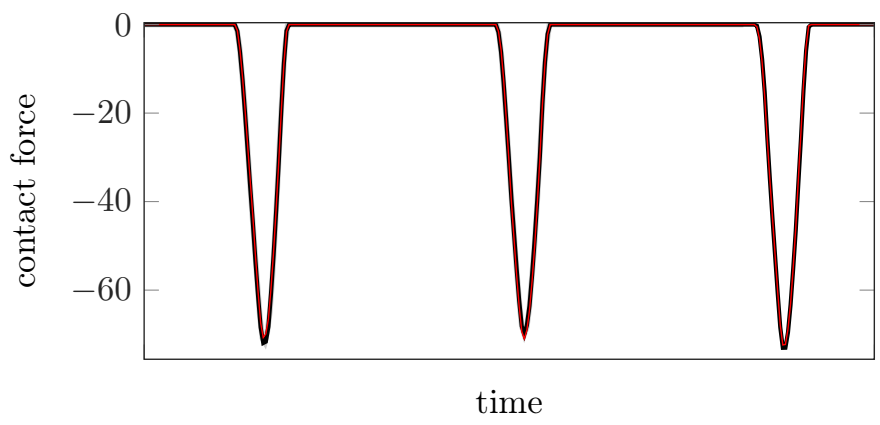

(b) radial contact force

Figure 9. Superimposition of results obtained with a NLC-ROM $\left(N_{p}=3\right)$ for different values of $h$ : $h=10^{-7} \mathrm{~s}(\square), h=5 \cdot 10^{-8} \mathrm{~s}(\longrightarrow)$ and $h=2.5 \cdot 10^{-8} \mathrm{~s}(\square)$.

obtained for different time steps are perfectly superimposed thus underlining the convergence of the numerical time integration procedure when a NLC-ROM is employed.

Similar results are obtained when increasing the reduction parameter $\eta$ or refining the spatial discretization of the abradable coating. These results are not detailed in this article for the sake of brevity. 


\section{Wear maps}

A wear map depicts the juxtaposition of the final wear profile on the casing predicted at the end of a simulation for each angular speed. The accuracy of these maps is thus directly related to the number of simulations (i.e. of angular speeds) carried out over the investigated angular speed range. These maps are plotted for two casing locations: in front of the blade leading edge (LE) and trailing edge (TE). For both a ROM and a NLC-ROM, two series of simulations are performed. (1) A first batch of results (see Figs. 10 and 11) comes from simulations with a ROM and a NLC-ROM as presented above: inertial effects impact both the blade stiffness and the blade/casing clearance configuration. (2) The second batch of results (see Figs. 12 and 13) has been obtained from simulations for which blade/casing clearances are independent on inertial effects. Accordingly, for these simulations, inertial effects only impact the blade stiffness. In the following. all wear maps feature the same colour code: from white (no wear) to black (maximum wear level: the $5 \mathrm{~mm}$ thick abradable layer is locally fully removed).

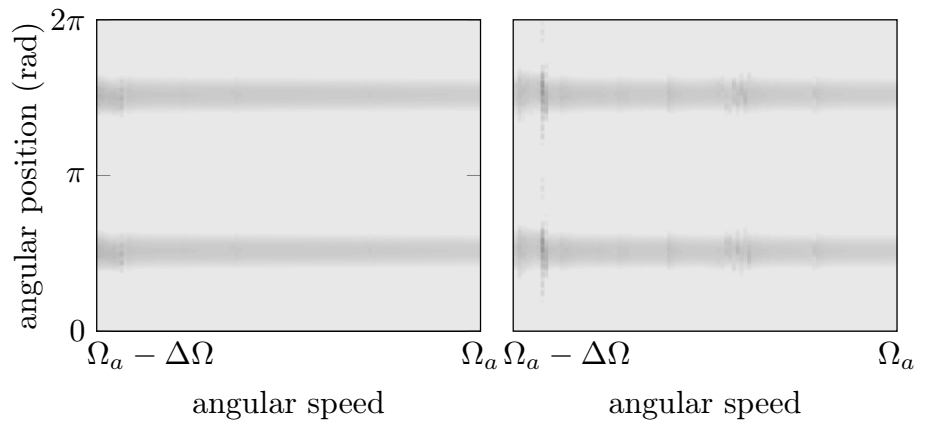

(a) ROM

(b) NLC-ROM

Figure 10. Wear maps in front of the leading edge

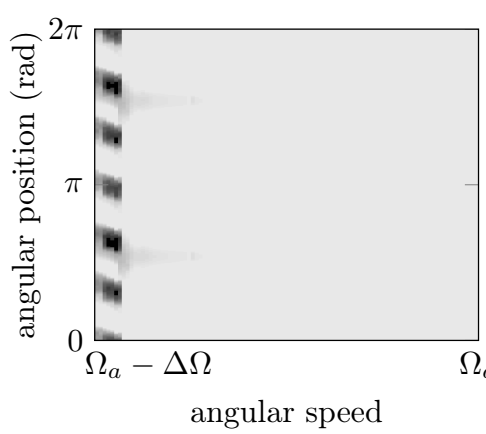

(a) ROM

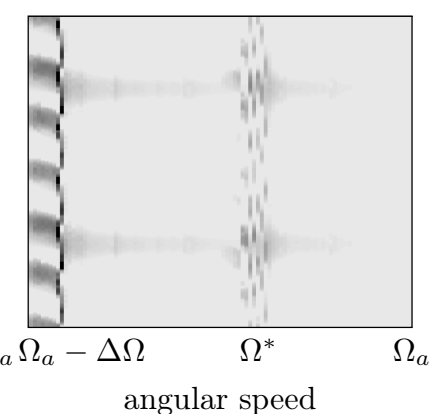

(b) NLC-ROM

Figure 11. Wear maps in front of the trailing edge

The wear maps plotted in Figs. 10 and 11 underline that over the considered angular speed range, there is no major wear predicted in front of the blade's leading edge. Indeed, both maps in Fig. 10 are very similar and do not feature any significant wear level. On the contrary, looking at the predicted wear levels in front of the blade's trailing edge in Fig. 11, specific wear patterns are detected. In particular, for $\Omega \simeq \Omega_{a}-\Delta \Omega$, six deep lobes are predicted both with a ROM and a NLC-ROM. A clear distinction can be made between the results obtained with a ROM and a NLC-ROM based on Fig. 11. As a matter of fact, it can be seen that for high angular speeds, there is no wear predicted in front of the trailing edge with a ROM. However, identical simulations with a NLC-ROM yield significant wear and even specific wear patterns (see, $\Omega \simeq \Omega^{*}$ in Fig. 11). These first results confirm the critical role that plays the accurate prediction of blade/casing clearance configurations for the prediction of rotor/stator interactions: it is evidenced that the approximation on blade/casing clearance configurations induced by a ROM may filter certain interactions. 
In order to assess more specifically the differences between a ROM and a NLC-ROM with respect to the blade's stiffness, wear maps pictured in Figs. 12 and 13 stem from simulations for which blade/casing clearance configurations are fixed and independent from inertial effects. This second batch of results thus allows to assess the role of the blade

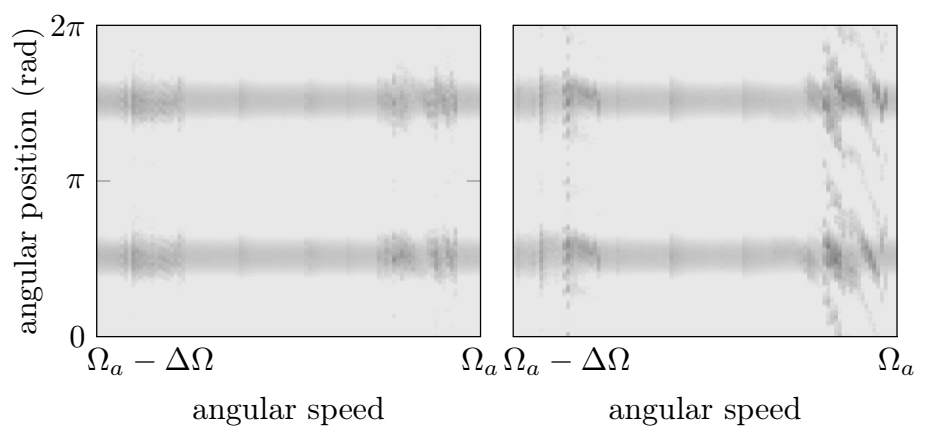

(a) ROM

(b) NLC-ROM

Figure 12. Wear maps in front of the leading edge, inertial effects only act on the blade's stiffness

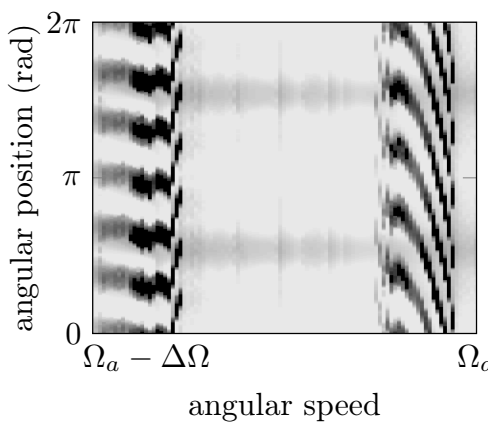

(a) ROM

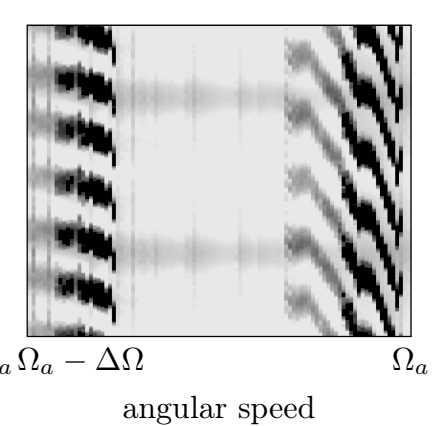

(b) NLC-ROM

Figure 13. Wear maps in front of the trailing edge, inertial effects only act on the blade's stiffness

stiffness matrix $\mathbf{K}(\Omega)$ in the detection of critical interactions. Results pictured in Figs. 12 and 13 underline that for both the ROM and the NLC-ROM, two interactions are predicted over the angular speed range $\Omega \in\left[\Omega_{a}-\Delta \Omega ; \Omega_{a}\right]$ : a six-lobe interaction is predicted for lower speeds while a five-lobe interaction is found for higher speeds. In both cases, wear lobes are essentially visible in front of the blade trailing edge, see Fig. 13. Only low wear levels are predicted in front of the leading edge, see Fig. 12. Though more complex wear patterns are observed in front of the leading edge with a NLC-ROM for high angular speeds, more in-depth analysis of the blade time response would be required to confirm a distinct vibration behaviour between the ROM and the NLC-ROM. Indeed, these lobes may very well be related to the blade's transient response which amplitude may vary from the ROM to the NLC-ROM. Overall, results obtained with a ROM and a NLC-ROM are very close when inertial effects do not affect the blade/casing clearance configuration.

In the end, results presented in this section highlight that the NLC-ROM provides significant improvements to the usual ROM allowing for a much more precise prediction of blade/casing clearance configurations. The enriched polynomial interpolation of the stiffness yields consistent results with those obtained with a ROM. More differences are to be expected in the case of blades more sensitive to inertial effects such as fan blades.

\section{CONCLUSION}

This article focuses on the presentation of an enriched reduced-order model of bladed components within turbomachines. It aims at accurately capturing the impact of inertial effects on both the component's stiffness anf the blade/casing clearance configurations. It is 
thoroughly validated by investigating the convergence of the proposed formulation as well as its applicability for the numerical prediction of rotor/stator interactions stemming from structural contacts along the blade-tip. It is demonstrated that the proposed reduced-order model yields a much more accurate prediction of blade-tip/casing clearances, a quantity for which usual linear formulations may fail to provide accurate predictions. The quality of the obtained results (in terms of eigenfrequencies and clearance configurations) is also underlined with respect to full finite element computations. However, the proposed method must be employed carefully. Indeed, attention must be paid to the numerical sensitivity of the obtained reduced-order model, particularly with respect to the conditioning of reduced matrices. It is underlined that considering a too high degree of interpolation may yield ill-conditioned reduced matrices and inaccurate results. In order to avoid any numerical issue, a compromise must be found between the degree of interpolation and the width of the considered angular speed range. Nonetheless, the proposed methodology is applied successfully to the numerical prediction of rotor/stator interactions within a low-pressure compressor. Presented results confirm that accounting for a non-linear computation of inertial effects leads to more physically relevant reduced-order models: specific critical regimes that are predicted with the proposed method are filtered when using classical reduced-order models relying on a linear formulation of inertial effects. It should be underlined that the extension of the proposed approach to full bladed-disks is immediate.

\section{ACKNOWLEDGMENT}

This research was supported by the Natural Sciences and Engineering Research Council of Canada (NSERC). The authors are also grateful to the industrial partner for supporting this project, Safran Aircraft Engines.

\section{References}

[1] Zilli, A., Williams, R., and Ewins, D., 2015. "Nonlinear dynamics of a simplified model of an overhung rotor subjected to intermittent annular rubs". Journal of Engineering for Gas Turbines and Power, 137, pp. 065001-1-10.

[2] Williams, R. J., 2011. "Simulation of blade casing interaction phenomena in gas turbines resulting from heavy tip rubs using an implicit time marching method". In Proceedings of the ASME Turbo Expo 2011 conference, GT2011-45495.

[3] Padova, C., Dunn, M. G., Barton, J., Turner, K., Turner, A., and DiTommaso, D., 2011. "Casing treatment and blade-tip configuration effects on controlled gas turbine blade tip/shroud rubs at engine conditions". Journal of Turbomachinery, 133(1), p. 011016.

[4] Ma, H., Wang, D., Tai, X., and Wen, B., 2015. "Vibration response analysis of blade-disk dovetail structure under blade tip rubbing condition". Journal of Vibration and Control.

[5] Batailly, A., Meingast, M., and Legrand, M., 2015. "Unilateral contact induced blade/casing vibratory interactions in impellers: analysis for rigid casings". Journal of Sound and Vibration, 337, pp. 244-262.

[6] Sternchüss, A., and Balmès, E., 2006. "On the reduction of quasi-cyclic disks with variable rotation speeds". Proceedings of the International Conference on Advanced Acoustics and Vibration Engineering (ISMA), pp. 3925-3939.

[7] Batailly, A., and Millecamps, A., 2016. "Minimizing clearance consumption: a key factor for the design of blade robust to rotor/stator interactions ?". In Proceedings of the ASME Turbo Expo 2016 conference, GT2016-56721.

[8] Batailly, A., Legrand, M., Millecamps, A., and Garcin, F., 2015. "Conjectural bifurcation analysis of the contact-induced vibratory response of an aircraft engine blade". Journal of Sound and Vibration, 348, pp. 239-262.

[9] Erler, E., Vo, H. D., and Yu, H., 2015. "Desensitization of axial compressor performance and stability to tip clearance size". In Proceedings of the ASME Turbo Expo 2015 conference, GT2015-42746. 
[10] Craig, R., and Bampton, M., 1968. "Coupling of substructures for dynamic analyses". AIAA Journal, 6(7), pp. 1313-1319.

[11] Bladh, R., Castanier, M. P., and Pierre, C., 2001. "Component-mode-based reduced order modeling techniques for mistuned bladed disks - part i: theoretical models". Journal of Engineering for Gas Turbines ans Power, 123(4), pp. 89-99.

[12] Rixen, D.J., 2009. "Dual craig-bampton with enrichment to avoid spurious modes". In Proceedings of the IMAC-XXVII conference.

[13] Allemang, R., and Brown, D., 1982. "A correlation coefficient for modal vector analysis". In Proceedings of the $1^{\text {st }}$ International Modal Analysis Conference, pp. 110-116.

[14] Millecamps, A., Batailly, A., Legrand, M., and Garcin, F., 2015. "Snecma's viewpoint on the numerical and experimental simulation of blade-tip/casing unilateral contacts". In Proceedings of the ASME Turbo Expo 2015 conference, GT2015-42682. 